\title{
Purification, crystallization and X-ray crystallographic analysis of methylmalonyl- CoA epimerase from Metallosphaera sedula
}

\author{
Seongjoon Joo, Seul Hoo Lee, Donghoon Lee and Kyung-Jin Kim* \\ School of Life Sciences, BK21 FOUR KNU Creative BioResearch Group, KNU Institute for Microorganisms, Kyungpook National \\ University, Daegu 41566, Republic of Korea \\ *Correspondence: kkim@knu.ac.kr
}

Methylmalonyl-CoA epimerase (MMCE) is an enzyme involved in the carbon capturing 3-HP/4-HB cycle, by catalyzing the reaction of converting (S)-methylmalonyl-CoA to (R)-methylmalonyl-CoA. In this study, we overexpressed MMCE from Metallosphaera sedula (MsMMCE) and purified the protein to homogeneity by Ni-NTA affinity and size-exclusion chromatography. The MsMMCE protein was crystallized using the hanging-drop vapor-diffusion method in the presence of $20 \%(\mathrm{w} / \mathrm{v})$ polyethylene glycol 3350 , and $0.2 \mathrm{M}$ ammonium nitrate at $293 \mathrm{~K}$. X-ray diffraction data were collected to a maximum resolution of $2.1 \AA$. The MsMMCE crystals belong to the space group $P 2_{1}$ with unit cell parameters $a=51.87 \AA, b=$ $79.00 \AA, c=106.09, \alpha=\gamma=90.0^{\circ}, \beta=95.80^{\circ}$. With six molecules of MsMMCE per an asymmetric unit, the crystal volume per unit of protein mass is $2.33 \AA^{3} \mathrm{Da}^{-1}$, which corresponds to a solvent content of approximately $47.13 \%$.

\section{INTRODUCTION}

Accumulation of carbon dioxide $\left(\mathrm{CO}_{2}\right)$ in the atmosphere due to continued use of fossil fuels after the Industrial Revolution has caused environmental problems such as global climate change. Many countries are working towards carbon neutrality by reducing carbon emissions, such as reducing the use of fossil fuels (Arctic Climate Impact Assessment, 2004; McGuire et al., 2006; McGuire et al., 2009). There is also an increasing interest in microbes that can capture $\mathrm{C} 1$ carbon. In prokaryotes, a total of six distinct autotrophic $\mathrm{CO}_{2}$ fixation pathway have been identified, such as the reductive pentose phosphate cycle (Pirson, 1960), the reductive citric acid cycle (Antranikian et al., 1982; Shiba et al., 1985), the reductive acetyl-CoA pathway (Pezacka and Wood, 1986), the 3-hydroxypropionate/malyl-CoA cycle (Strauss and Fuchs, 1993), the dicarboxylate/4-hydroxybutyrate cycle (Huber et al., 2008), and the 3-hydroxypropionate/4hydroxybutyrate (3-HP/4HB) cycle (Berg et al., 2007).

The Metallosphaera sedula identified in the volcanic zone of Pisciarelli Solfatara in Naples, Italy is the first strain known to be capable of carbon fixation through the 3-HP/4-HB cycle (Huber et al., 1989; Berg et al., 2007; Berg et al., 2010; Estelmann et al., 2011). The 3-HP/4-HB cycle can be largely divided into two sub-pathways, a 3-HP sub-pathway and a 4-HB sub-pathway. The 3-HP sub-pathway begins when acetyl-CoA is converted to malonyl-CoA by capturing carbonate enzymatically (Chuakrut et al., 2003; Hügler et al., 2003; Berg et al., 2007; Teufel et al., 2009; Estelmann et al., 2011). This malonyl-CoA is converted to propionyl-CoA through 5 enzymatic reactions (Chuakrut et al., 2003; Hügler et al., 2003; Berg et al., 2007; Teufel et al., 2009; Estelmann et al., 2011). Then, propionyl-CoA is converted to (S)-methylmalonyl-CoA through an enzymatic reaction that captures carbonate once again (Chuakrut et al., 2003; Hügler et al., 2003; Berg et al., 2007; Teufel et al., 2009; Estelmann et al., 2011). Thereafter, (S)-methylmalonyl-CoA is converted to (R)methylmalonyl-CoA by methylmalonyl-CoA epimerase (MMCE), and finally becomes succinyl-CoA by methylmalonyl-CoA mutase (Chuakrut et al., 2003; Hügler et al., 2003; Berg et al., 2007; Teufel et al., 2009; Estelmann et al., 2011). In this way, acetylCoA was converted to succinyl-CoA with two more carbons by receiving two molecules of carbonate through the 3-HP subpathway (Chuakrut et al., 2003; Hügler et al., 2003; Berg et al., 2007; Teufel et al., 2009; Estelmann et al., 2011). Subsequently, succinyl-CoA is divided into two acetyl-CoA through seven enzymatic reactions in the 4-HB sub-pathway (Chuakrut et al., 2003; Hügler et al., 2003; Berg et al., 2007; Teufel et al., 2009; Estelmann et al., 2011).

In general, MMCE is involved in the process of converting 3-carbon propionyl-CoA into 4-carbon succinyl-CoA in the lipolysis pathway of an odd numbered carbon chain lipid (Chalmers and Lawson, 1982). When propionyl-CoA is converted to methylmalonyl-CoA by propionyl-CoA carboxylase, it takes the S-form (Chalmers and Lawson, 1982). Since methylmalonylCoA mutase recognizes only R-form methylmalonyl-CoA as a substrate and converts it to succinyl-CoA, chiral conversion of S-form methylmalonyl-CoA to R-form is essential (Chalmers and Lawson, 1982). MMCE is an enzyme that catalyzes this chiral conversion of (S)-methylmalonyl-CoA to (R)-methylmalonyl-CoA (Figure 1) (Chalmers and Lawson, 1982).

In $M$. sedula, the same reaction from propionyl-CoA to succinyl-CoA occurs in the 3-HP sub-pathway during the 
3-HP/4-HB carbon fixation cycle (Teufel et al., 2009). Therefore, MMCE from $M$. sedula (MsMMCE) is an essential enzyme for carbon fixation of $M$. sedula. Despite this importance, research on MsMMCE is insufficient. Thus, in this study, we described the cloning, expression, purification, crystallization, and X-ray crystallographic analysis of the MsMMCE protein.

\section{MATERIALS AND METHODS}

\section{Production of MsIMIME}

We designed the forward and reverse primers as 5'-GCGC CATATGGAAACCTT AGATATAGATCACGTG-3' and 5'-GCGC CTCGAGATGGGTTTCTTGAACGAGTTCCA G-3' with introduced Ndel and Xhol restriction sites, respectively (underlined). The MsMMCE gene was amplified by polymerase chain reaction (PCR) using genomic DNA from $M$. sedula as a template. The PCR product was then subcloned into pET30a expression vector (Novagen) with a six-His tag at the C-terminus. The PET30a:MsMMCE was transformed into the $E$. coli strain $\mathrm{BL21}(\mathrm{DE} 3)-\mathrm{T} 1^{\mathrm{R}}$, which was grown in $1 \mathrm{~L}$ of Luria-Bertani medium containing kanamycin at $37^{\circ} \mathrm{C}$. At an $\mathrm{OD}_{600}$ of 0.6 , MsMMCE protein expression was induced by addition of $0.5 \mathrm{mM}$ isopropyl $\beta$-D-1-thiogalactopyranoside (IPTG) and the culture medium was maintained for a further $16 \mathrm{~h}$ at $18^{\circ} \mathrm{C}$. the culture was then harvested by centrifugation at $4,000 \times g$ for $15 \mathrm{~min}$ at $20^{\circ} \mathrm{C}$. The cell pellet was resuspended in buffer A $(40 \mathrm{mM}$ Tris- $\mathrm{HCl}$, $\mathrm{pH}$ 8.0) and then disrupted by ultrasonication. The cell debris was removed by centrifugation at $13,500 \times g$ for $30 \mathrm{~min}$. And supernatant was applied to an Ni-NTA agarose column (Thermo Fisher Scientific, USA). After washing with buffer A containing $30 \mathrm{mM}$ imidazole, the bound proteins were eluted with $300 \mathrm{mM}$ imidazole in buffer A. Finally, a trace number of contaminants<smiles>C[C@H](C(=O)O)C(=O)SC(=O)O</smiles>

(S)-methylmalonyl-CoA

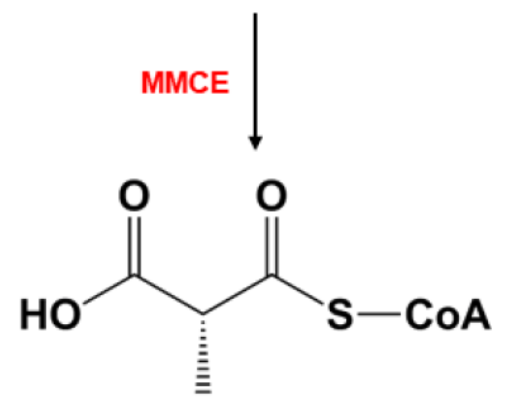

(R)-methylmalonyl-CoA

FIGURE 1 I Enzyme reaction of MsMMCE. were removed by size-exclusion chromatography by using a Hiprep 26/60 Sephacryl S-300 HR column (GE Healthcare Life Sciences) equilibrated with buffer $A$. We performed purification experiments at $4^{\circ} \mathrm{C}$. SDS-PAGE analysis of the purified proteins shows a single polypeptide of $15.5 \mathrm{kDa}$ corresponding to the molecular weight of the MsMMCE monomer. The purified protein was concentrated to $135 \mathrm{mg} / \mathrm{ml}$ in $40 \mathrm{mM}$ Tris- $\mathrm{HCl}, \mathrm{pH} 8.0$. Macromolecule-production information is given in Table 1.

\section{Crystallization of MsMMCE}

Crystallization of the purified MsMMCE was initially performed with commercially available spares-matrix screens, including Index, PEG ion I and II (Hampton Research), and Wizard Classic I and II (Rigaku Reagents), using the hanging-drop vapordiffusion method at $20^{\circ} \mathrm{C}$. Each experiment consisted of mixing $1.0 \mu \mathrm{L}$ protein solution (135 mg/ml in $40 \mathrm{mM}$ Tris- $\mathrm{HCl}, \mathrm{pH} 8.0$ ) with $1.0 \mu \mathrm{L}$ reservoir solution and then equilibrating this against $500 \mu \mathrm{L}$ reservoir solution. MsMMCE crystals were observed from several crystallization conditions. After several steps for crystal improvement, the best quality crystals appeared in $20 \%$ $(\mathrm{w} / \mathrm{v})$ polyethylene glycol 3350 , and $0.2 \mathrm{M}$ ammonium nitrate. Crystallization information is summarized in Table 2.

\section{X-ray diffraction analysis of MsIMMCE}

Diffraction data from MsMMCE crystals were collected at the 7A beamline of the Pohang Accelerator Laboratory (PAL, Pohang, Korea) (Park et al., 2017), using a Quantum 270 CCD detector (ADSC, USA). The crystals of MsMMCE diffracted to a resolution of $2.1 \AA$. All data were indexed, integrated, and scaled using the HKL-2000 software package (Otwinowski and Minor, 1997). The data statistics are summarized in Table 3.

\section{RESULTS AND DISCUSSION}

MsMMCE protein was purified by Ni-NTA affinity chromatography and then further purified through size-exclusion chromatography to obtain purified single protein. The eluted protein from size-

TABLE 1 I Macromolecule-production information

\begin{tabular}{ll}
\hline Source organism & Metallosphaera sedula \\
\hline DNA source & Chromosomal DNA \\
\hline Forward primer & GCGCCATATGGAAACCTTAGATATAGATCACGTG \\
\hline Reverse primer & GCGCCTCGAGATGGGTTCTTGAACGAGTTCCAG \\
\hline Cloning vector & pET-30a \\
\hline Expression vector & pET-30a \\
\hline Expression host & E. coli BL21 (DE3)-T1 \\
\hline & METLDIDHVGVAVENLEEAIKLYTEKMGMKL \\
Complete amino-acid & VHREDLPDR GIKVAFLTGNEGTTAVELMEP \\
sequence of the & MNHEDPNNTVAKFLKTRGQ GMHHLAVKV \\
construct produced & KDINSSLRDLEGKGLTLIDKNGRKGARGHLV \\
& AFVHPKSVMGLLLEVQETHLEHHHHHH \\
\hline
\end{tabular}

The underlined characters in the primers indicate restriction-enzyme sites. The bold-face characters in the complete amino-acid sequence of the construct produced indicate C-terminal tag. 
TABLE 2 I Crystallization

\begin{tabular}{ll}
\hline Method & Hanging-drop vapor diffusion \\
\hline Plate type & SPL plate (24-well) \\
\hline Temperature $(\mathrm{K})$ & 293 \\
\hline Protein concentration $\left(\mathrm{mg} \mathrm{m}^{-1}\right)$ & 135 \\
\hline $\begin{array}{l}\text { Buffer composition of } \\
\text { protein solution }\end{array}$ & $40 \mathrm{mM}$ Tris-HCl pH 8.0 \\
\hline Composition of reservoir solution & $\begin{array}{l}20 \% \text { polyethylene glycol 3350, and } \\
0.2 \mathrm{M} \text { Ammonium nitrate }\end{array}$ \\
\hline Volume and ratio of drop & $\begin{array}{l}2 \mu \mathrm{L} ; 1: 1 \text { ratio of protein and } \\
\text { reservoir solutions }\end{array}$ \\
\hline Volume of reservoir $(\mathrm{ml})$ & 0.5 \\
\hline
\end{tabular}

TABLE 3 I Data collection and processing

\begin{tabular}{|c|c|}
\hline Diffraction source & Beamline 7A, PAL \\
\hline Wavelength $(\AA ̊)$ & 0.97934 \\
\hline Temperature (K) & 100 \\
\hline Detector & Quantum 270 CCD \\
\hline Crystal-to-detector distance (mm) & 200 \\
\hline Rotation range per image $\left(^{\circ}\right)$ & 1 \\
\hline Total rotation range $\left({ }^{\circ}\right)$ & 360 \\
\hline Exposure time per image(s) & 1 \\
\hline Space group & $P 2_{1}$ \\
\hline$a, b, c(\AA)$ & $51.9,79.0,106.1$ \\
\hline$\alpha, \beta, \gamma\left({ }^{\circ}\right)$ & $90.0,95.8,90.0$ \\
\hline Resolution range (Å) & $50.00-2.05(2.09-2.05)^{a}$ \\
\hline Total No. of reflections & 54070 \\
\hline Completeness (\%) & $97.2(95.7)^{a}$ \\
\hline Rsym or Rmerge (\%) & $4.7(30.4)^{\mathrm{a}}$ \\
\hline$|/ \sigma|$ & $20.8(2.5)^{a}$ \\
\hline Redundancy & $3.1(2.5)^{\mathrm{a}}$ \\
\hline
\end{tabular}

aThe numbers in parentheses are statistics from the highest resolution shell.

exclusion chromatography column, calibrated with Gel Filtration Calibration Kits (GE Healthcare Life Sciences), had a molecular weight of $\sim 31 \mathrm{kDa}$ which match a dimer form of MsMMCE protein (data not shown). SDS-PAGE of the purified MsMMCE protein showed a single band with a monomeric molecular weight of $15.5 \mathrm{kDa}$ (Figure 2).

The MsMMCE protein was crystallized in $20 \%(\mathrm{w} / \mathrm{v})$ polyethylene glycol 3350, and 0.2 M ammonium nitrate (Figure 3). The crystals of MsMMCE protein were moved to cryo protectant solution containing $26 \%(\mathrm{w} / \mathrm{v}$ ) polyethylene glycol 3350, $0.2 \mathrm{M}$ ammonium nitrate, and $30 \%(\mathrm{v} / \mathrm{v})$ glycerol. The crystals were fished out with a loop larger than the crystals, and flash-frozen by immersion in liquid nitrogen. The crystals of MsMMCE protein diffracted to resolution of $2.1 \AA$. The MsMMCE crystals belonged to the space group $P 2_{1}$ with unit cell parameters $a=51.87 \AA, b$ $=79.00 \AA, c=106.09 \AA, \alpha=\gamma=90.0^{\circ}, \beta=95.80^{\circ}$. Assuming six molecules of MsMMCE protein (15.5 kDa) per asymmetric unit,

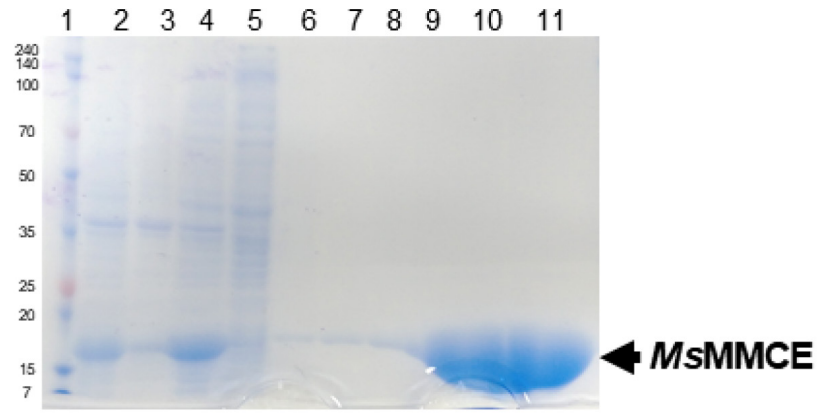

FIGURE 2 I Purification of MsMMCE. SDS-PAGE of purification of recombinant MsMMCE protein. Lane 1 shows molecular-weight markers (labelled in $\mathrm{kDa}$ ). Lanes 2-11 show the purification procedure of MsMMCE using Ni-NTA chromatography. Lane 2, whole cell extract; lanes 3 and 4, pellet and supernatant fraction after centrifugation of whole cell extract, respectively; lane 5, flowthrough from Ni-NTA column; lane 6 to 9, wash with $0,10,20$, and $30 \mathrm{mM}$ imidazole, respectively; lane 10 and 11, elution with $300 \mathrm{mM}$ imidazole. Purified MsMMCE is indicated with an arrow.

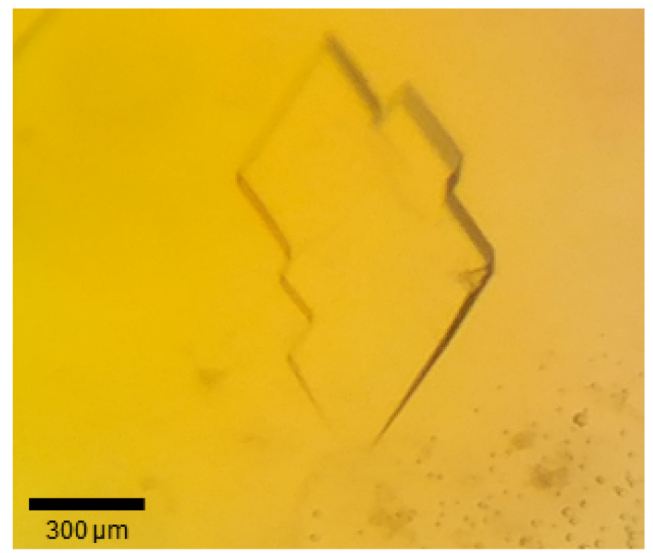

FIGURE 3 I Crystals of MsMMCE. The best quality crystal was produced from the condition with $20 \%$ polyethylene glycol 3350 , and $0.2 \mathrm{M}$ ammonium nitrate and grew to maximum dimensions of $0.2 \times 0.2 \times 0.05$ $\mathrm{mm}$ within 3 days.

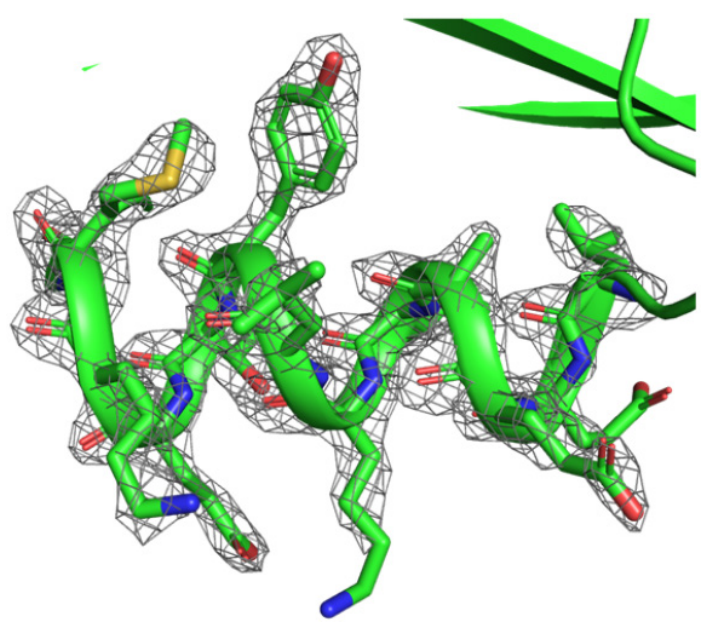

FIGURE 4 I Initial 2Fo-Fc maps of MsMMCE structure. The $2 F_{0}-F_{c}$ maps (contoured at $1.0 \sigma$ ) are shown as gray meshes. 
the crystal volume per unit of protein mass was $2.33 \AA^{3} \mathrm{Da}^{-1}$, meaning that the solvent content was approximately $47.13 \%$ (Matthews, 1968).

We determined the structure of MsMMCE protein by molecular replacement with the CCP4 version of MOLREP (Vagin and Teplyakov, 1997) using the structure of hypothetical protein $\mathrm{BH} 1468$ from Bacillus halodurans C-125 (BhHP) (PDB CODE 3OA4) as a search model. MsMMCE shows $38.64 \%$ amino acid sequence identity to BhHP. We performed model building and refinement using the program WinCoot (Emsley and Cowtan, 2004) and CCP4 refmac5 (Murshudov et al., 1997), respectively. The initial electron density map, which was good quality with backbone atoms well defined by electron density, allowed us to build a three-dimensional structure of MsMMCE (Figure 4). Crystallographic model building and structure refinement to 2.1 Å resolution are in progress.

\section{ACKNOWLEDGEMENTS}

This work was supported by the Technology Development Program to Solve Climate Changes of the National Research Foundation (NRF) funded by the Ministry of Science and ICT (NRF-2017M1A2A2087631).

\section{CONFLICT OF INTEREST}

The authors have declared that no competing interest exist.

Original Submission: May 25, 2021

Revised Version Received: Jun 10, 2021

Accepted: Jun 16, 2021

\section{REFERENCES}

Antranikian, G., Herzberg, C., and Gottschalk, G. (1982). Characterization of ATP citrate lyase from Chlorobium limicola. $J$ Bacteriol 152, 12841287.

Arctic Climate Impact Assessment. Impacts of a warming Artic (Cambridge University Press, 2004).

Berg, I.A., Kockelkorn, D., Buckel, W., and Fuchs, G. (2007). A 3-hydroxypropionate/4-hydroxybutyrate autotrophic carbon dioxide assimilation pathway in Archaea. Science 318, 1782-1786.

Berg, I.A., Ramos-Vera, W.H., Petri, A., Huber, H., and Fuchs, G. (2010). Study of the distribution of autotrophic $\mathrm{CO} 2$ fixation cycles in Crenarchaeota. Microbiology (Reading) 156, 256-269.

Chalmers, R.A., and Lawson, A.M. Organic acids in man Ch.11 (Springer, 1982).

Chuakrut, S., Arai, H., Ishii, M., and Igarashi, Y. (2003). Characterization of a bifunctional archaeal acyl coenzyme A carboxylase. J Bacteriol 185, 938-947.
Emsley, P., and Cowtan, K. (2004). Coot: model-building tools for molecular graphics. Acta Crystallogr D Biol Crystallogr 60, 2126-2132.

Estelmann, S., Hügler, M., Eisenreich, W., Werner, K., Berg, I.A., RamosVera, W.H., Say, R.F., Kockelkorn, D., Gad'on, N., and Fuchs, G. (2011). Labeling and enzyme studies of the central carbon metabolism in Metallosphaera sedula. J Bacteriol 193, 1191-1200.

Hügler, M., Krieger, R.S., Jahn, M., and Fuchs, G. (2003). Characterization of acetyl-CoA/propionyl-CoA carboxylase in Metallosphaera sedula. Carboxylating enzyme in the 3-hydroxypropionate cycle for autotrophic carbon fixation. Eur J Biochem 270, 736-744.

Huber, G., Spinnler, C., Gambacorta, A., and Stetter, K.O. (1989). Metallosphaera sedula gen, and $\mathrm{sp}$. nov. represents a new genus of aerobic, metal-mobilizing, thermoacidophilic archaebacteria. Syst Appl Microbiol 12, 38-47.

Huber, H., Gallenberger, M., Jahn, U., Eylert, E., Berg, I.A., Kockelkorn, D., Eisenreich, W., and Fuchs, G. (2008). A dicarboxylate/4-hydroxybutyrate autotrophic carbon assimilation cycle in the hyperthermophilic Archaeum Ignicoccus hospitalis. Proc Natl Acad Sci U S A 105, 7851-7856.

Matthews, B.W. (1968). Solvent content of protein crystals. J Mol Biol 33 , 491-497.

McGuire, A.D., Anderson, L.G., Christensen, T.R., Dallimore, S., Guo, L., Hayes, D.J., Heimann, M., Lorenson, T.D., Macdonald, R.W., and Roulet, N. (2009). Sensitivity of the carbon cycle in the Arctic to climate change. Ecol Monogr 79, 523-555.

McGuire, A.D., III, F.S.C., Walsh, J.E., and Wirth, C. (2006). Integrated regional changes in Arctic climate feedbacks: implications for the global climate system. Annu Rev Environ Resour 31, 61-91.

Murshudov, G.N., Vagin, A.A., and Dodson, E.J. (1997). Refinement of macromolecular structures by the maximum-likelihood method. Acta Crystallogr D Biol Crystallogr 53, 240-255.

Otwinowski, Z., and Minor, W. (1997). Processing of X-ray diffraction data collected in oscillation mode. Methods Enzymol 276, 307-326.

Park, S.Y., Ha, S.C., and Kim, Y.G. (2017). The protein crystallography beamlines at the Pohang Light Source II. Biodesign 5, 30-34.

Pezacka, E., and Wood, H.G. (1986). The autotrophic pathway of acetogenic bacteria. Role of $\mathrm{CO}$ dehydrogenase disulfide reductase. $J$ Biol Chem 261, 1609-1615.

Pirson, A. Die CO2-assimilation/the assimilation of carbon dioxide (Springer, 1960).

Shiba, H., Kawasumi, T., Igarashi, Y., Kodama, T., and Minoda, Y. (1985). The CO2 assimilation via the reductive tricarboxylic acid cycle in an obligately autotrophic, aerobic hydrogen-oxidizing bacterium, Hydrogenobacter thermophilus. Arch Microbiol 141, 198-203.

Strauss, G., and Fuchs, G. (1993). Enzymes of a novel autotrophic CO2 fixation pathway in the phototrophic bacterium Chloroflexus aurantiacus, the 3-hydroxypropionate cycle. Eur J Biochem 215, 633-643.

Teufel, R., Kung, J.W., Kockelkorn, D., Alber, B.E., and Fuchs, G. (2009). 3-hydroxypropionyl-coenzyme A dehydratase and acryloyl-coenzyme A reductase, enzymes of the autotrophic 3-hydroxypropionate/4hydroxybutyrate cycle in the Sulfolobales. J Bacteriol 191, 4572-4581.

Vagin, A., and Teplyakov, A. (1997). MOLREP: an automated program for molecular replacement. J Appl Cryst 30, 1022-1025. 